\title{
Inteligencia emocional y rendimiento académico en estudiantes de la Universidad Continental, Huancayo
}

\section{Emotional intelligence and academic performance in students of psychology of the Universidad Continental, Huancayo}

\section{RESUMEN}

El objetivo fue determinar la relación entre la inteligencia emocional y el rendimiento académico de los estudiantes de la Escuela de Psicología de la Universidad Continental. El trabajo fue transeccional correlacional, en una población de 246 estudiantes del primer semestre, siendo la muestra censal. Se aplicó el inventario de Bar-On (ICE), y los datos se procesaron con media aritmética, desviación estándar, prueba de "rho" de Spearman y Chi cuadrada. En la dimensión intrapersonal y rendimiento académico el coeficiente de correlación es rho $=-0,029$, en la dimensión interpersonal y rendimiento académico el coeficiente de correlación rho=-0,009, en la dimensión adaptabilidad y rendimiento académico el coeficiente de correlación rho $=-0,057$, en la dimensión manejo de tensión y rendimiento académico el coeficiente de correlación rho $=-0,021$, en la dimensión estado de ánimo y rendimiento académico el coeficiente rho=0,066 . Los resultados contradictorios respecto a la correlación entre la Inteligencia emocional y rendimiento académico ocurren porque hasta el momento no existe consenso entre qué es la Inteligencia Emocional, ya que existen enfoques diversos; unos que se decantan por entender la IE como una inteligencia genuina y otros como un modelo mixto que tiene una visión más orientada a variables de personalidad. Se concluye que no existe relación directa ni significativa entre las variables inteligencia emocional y rendimiento académico en los estudiantes de la carrera profesional de Psicología de la Universidad Continental.

Palabras Clave: Inteligencia emocional, rendimiento académico, adaptabilidad.

\section{ABSTRACT}

The objective was to determine the relationship between emotional intelligence and academic achievement of the students of the School of Psychology of the Universidad Continental. The work was correlational transactional, in a population of 246 students in the first semester, being the census sample. Applied inventory of Bar-On (I-CE), and data were processed with arithmetic mean, standard deviation, test of "rho" of Spearman and Chi square. In the intrapersonal dimensión and academic performance the correlation coefficient is rho $=-0,029$, in the interpersonal dimensión and academic performance the correlation coefficient rho $=-0,009$, in the dimensión adaptability and academic performance the correlation coefficient rho $=-0,057$, in the management of stress and academic achievement dimensión the coefficient of correlation rho $=0,021$, in the dimensión status mood and academic performance coefficient rho $=-0,066$. The conflicting results regarding the correlation between emotional intelligence and academic performance occur because so far there is no consensus between what is emotional intelligence, since there are different approaches; some who choose to undertand the El as a genuine intelligence and others as a mixed model that has a vision that is more oriented to personality variables. It is concluded that direct or significant relationship between the emotional intelligence and academic achievement in the students of the profesional career of Psychology of the Universidad Continental.

Keywords: Emotional intelligence, academic performance, adaptability.

Historial del artículo:

Recibido, 24 de mayo de 2017; aceptado, 10 de junio de 2017; disponible en línea, 25 de junio de 2017

* Psicólogo, docente de la Universidad Continental.

Correo: jsalcedo@continental.edu. 


\section{INTRODUCCIÓN}

La investigación fue motivada en las observaciones del trabajo profesional en la enseñanza en el nivel superior en general y en la formación de futuros psicólogos en particular. La formación de Psicólogos en el Perú según Alarcón (2000), tiene una tradición de más de 50 años en el Perú. Los orígenes de la psicología como ciencia provienen de la filosofía. Asimismo, el carácter científico y tecnológico que tiene en la actualidad, demanda que el profesional psicólogo formado en los centros de educación superior tenga una formación integral, científica, social y humanística, según Asamblea Nacional de Rectores (2006).

En ese sentido la formación de los psicólogos en las universidades del Perú, debe poner énfasis no solo en lo que compete a las habilidades procedimentales - cognitivas, sino sobre todo a las competencias actitudinales, como el trabajo en equipo, las habilidades interpersonales, el manejo de la presión, la capacidad de empatía y resilencia. Todas estas competencias incluyen dimensiones de la inteligencia emocional.

Con la identificación del nivel de cociente emocional de los estudiantes de Psicología en relación al rendimiento académico, se podrán diseñar estrategias didácticas para enfatizar el desarrollo de habilidades blandas como la del trabajo en equipo o las habilidades interpersonales.

La identificación del nivel de cociente emocional de los estudiantes de psicología permitirá formar psicólogos con habilidades actitudinales como el trabajo en equipo el autoconocimiento, que beneficiarán a la comunidad en general ya que estos profesionales podrán intervenir de mejor manera en la promoción de la salud psicológica.

Existen habilidades más importantes que la cognitiva para lograr un buen rendimiento académico, habilidades como la capacidad para percibir, asimilar y comprender y regular las propias emociones, que ayudan a promover el éxito personal y profesional. Es necesario encontrar la relación entre la inteligencia emocional y el rendimiento académico para promover programas de intervención psicológica que pueda potencializar esta inteligencia.

Servirá de base para otras investigaciones, así como contribuirá en aumentar el conocimiento de la relación entre la inteligencia emocional y el rendimiento académico en estudiantes de Psicología.

Es así que los diseños curriculares de las universidades deben incluir no solo los aspectos cognitivos sino también los aspectos emocionales, e interaccionales, asimismo, todos estos aspectos influyen en el ajuste psicológico posterior y el desempeño laboral, como menciona Páez y Castaño (2015).

Los alumnos emocionalmente inteligentes presentan mejor autoestima ajuste y bienestar psicológico, satisfacción emocional e interpersonal, calidad de las relaciones interaccionales, apoyo social, y menor disposición para presentar comportamientos disruptivos agresivos o violentos, así como presentan menores grados de síntomas físicos, ansiedad y depresión. Todas esas habilidades incrementan el rendimiento académico de los estudiantes (Extremera y Rey, 2007).

Todas las habilidades antes mencionadas cobran una dimensión especial en la formación de los futuros psicólogos debido a que por la naturaleza particular de su ejercicio profesional necesitan poseer todo un conjunto de habilidades como: la empatía, la cordialidad, la autenticidad, la aceptación incondicional; habilidades que pertenecen a los factores que incluye la inteligencia emocional y que es necesario desarrollarlos durante su formación profesional.

Para direccionar la indagación se formuló el problema principal que: żexiste relación directa entre inteligencia emocional y el rendimiento académico en la asignatura de Fundamentos de la Psicología en los estudiantes del primer semestre de la escuela profesional de Psicología de la Universidad Continental? el objetivo general fue; determinar el grado de relación entre la inteligencia emocional y el rendimiento académico en la asignatura de Fundamentos de la Psicología de los estudiantes del primer semestre de la Escuela Académico Profesional de Psicología de la Universidad Continental.

\section{MATERIAL Y MÉTODOS}

El presente estudio es de tipo exploratorio. El nivel de investigación es transecsacional correlacional porque se describen relaciones entre dos variables en un momento determinado (Cancela, 2010). Mantiene como propósito recoger información de la realidad para enriquecer el conocimiento científico.

Nuestro trabajo adoptó como método general al método científico, que es el conjunto de pasos o procedimientos que permiten comprobar o rechazar hipótesis (Cancela, 2010).

Es un estudio correlacional, las hipótesis tienden a verificar la relación de variables a través de la covariación o asociación.

Responde al siguiente esquema: 


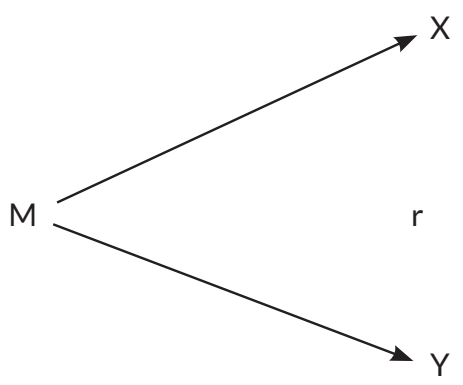

Donde:

M : Muestra

$X$ : Inteligencia emocional

$Y$ : Rendimiento académico

$r$ : Correlación

La población estuvo conformada por 246 estudiantes de la Escuela Profesional de Psicología de la Universidad Continental del primer semestre del curso de Fundamentos de la Psicología del período 2016-I, coincidiendo nuestra muestra con el universo por ser de carácter censal.

Se trabajó con 182 estudiantes del primer ciclo en el período académico antes mencionado debido a que 64 estudiantes no cumplieron con los criterios de inclusión.

Tabla 1

Población por secciones.

\begin{tabular}{lcc}
\hline Sección & $\begin{array}{c}\mathbf{N}^{\circ} \text { de estudiantes } \\
\text { matriculados }\end{array}$ & $\mathbf{N}^{\circ}$ de encuestados \\
\hline $\mathrm{BC} 1005$ & 42 & 30 \\
$\mathrm{BC} 1006$ & 39 & 28 \\
$\mathrm{BC} 1061$ & 44 & 38 \\
$\mathrm{BC} 1101$ & 39 & 25 \\
$\mathrm{BC} 1003$ & 49 & 37 \\
$\mathrm{BC} 1004$ & 33 & 24 \\
Total & 246 & 182 \\
\hline
\end{tabular}

Para conocer los niveles de inteligencia emocional en los estudiantes de la Escuela de Psicología, se utilizó como técnica la encuesta y como instrumento el cuestionario de ICE- BarOn, que consta de 133 ítems claros y comprensibles. El instrumento integra 5 componentes y 15 sub componentes cuya sumatoria dio como resultado la medida total de la inteligencia emocional. La escala de respuestas fue de tipo Likert con formato de respuestas de auto clasificación de cinco puntos.

El instrumento cuenta con validez de constructo en la medida que existe suficiente base teórica al respecto, evidenciando el grado en que la medición realizada se relaciona consistentemente con otras mediciones.

Para medir la confiabilidad del instrumento se realizó la Prueba Piloto, aplicando el instrumento a 30 estudiantes de la Escuela de Psicología de la Universidad Continental, cuyos resultados fueron procesados mediante el Coeficiente de Alfa de Crombach. El valor del coeficiente Alfa de Crombach que se obtuvo es de 0,930. Resultado que afirma que el instrumento es muy confiable, ya que el valor obtenido es mayor a 0,90 .

Para la comprobación de la hipótesis se ha empleado la prueba de una proporción con la normal o Z de Gauss, al $95 \%$ de confianza estadística.

Para determinar la relación de dichas variables primero realizamos la prueba de hipótesis de normalidad con la finalidad de determinar el estadístico de prueba que en nuestro caso fue la rho de Spearman, ilustrando las características de la relación con la figura de dispersión respectiva.

\section{RESULTADOS}

Se observa en los resultados que el mayor porcentaje (67\%) de estudiantes que respondieron el inventario de BarOn, tienen una inteligencia emocional adecuada, que se constituye en un promedio aceptable.

Otro dato para destacar es que el $16 \%$ de los estudiantes que respondieron el inventario tienen entre bajo y muy bajo su nivel de inteligencia emocional, que se constituye en el grupo de estudiantes que necesitan mejorar considerablemente su nivel de inteligencia emocional. Además, se observa que existe un $17 \%$ de estudiantes cuya inteligencia emocional se halla en los niveles alto y muy alto de inteligencia emocional, que implica una inteligencia emocional bastante desarrollada.

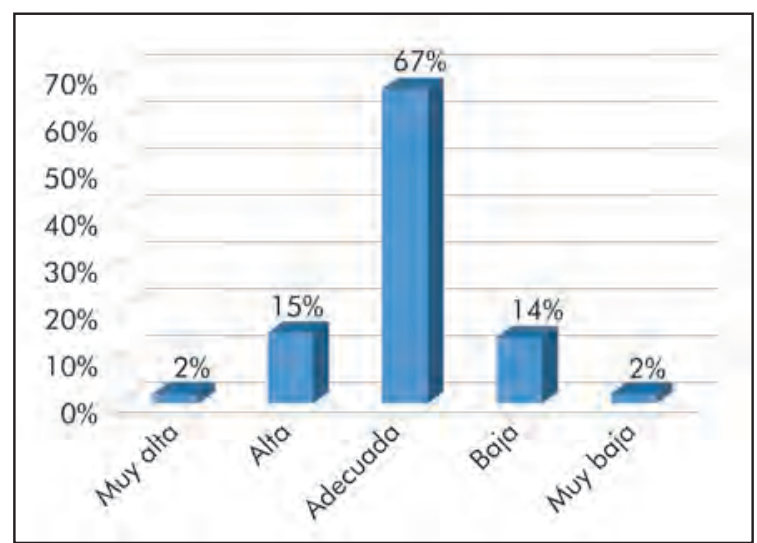

Figura 1. Niveles de inteligencia emocional. 
Tabla 2

Niveles de la inteligencia emocional, por dimensiones.

\begin{tabular}{|c|c|c|c|c|c|c|c|c|c|c|}
\hline \multirow[t]{2}{*}{ Niveles } & \multicolumn{2}{|c|}{ Intrapersonal } & \multicolumn{2}{|c|}{ Interpersonal } & \multicolumn{2}{|c|}{ Adaptabilidad } & \multicolumn{2}{|c|}{$\begin{array}{c}\text { Manejo de } \\
\text { tensión }\end{array}$} & \multicolumn{2}{|c|}{$\begin{array}{c}\text { Estado } \\
\text { de ánimo }\end{array}$} \\
\hline & Frec. & $\%$ & Frec. & $\%$ & Frec. & $\%$ & Frec. & $\%$ & Frec. & $\%$ \\
\hline Muy alta & 3 & $2 \%$ & 1 & $1 \%$ & 2 & $1 \%$ & 1 & $1 \%$ & 1 & $1 \%$ \\
\hline Alta & 22 & $15 \%$ & 28 & $19 \%$ & 17 & $12 \%$ & 19 & $13 \%$ & 20 & $14 \%$ \\
\hline Adecuada & 98 & $68 \%$ & 98 & $68 \%$ & 104 & $72 \%$ & 102 & $71 \%$ & 98 & $68 \%$ \\
\hline Baja & 17 & $12 \%$ & 14 & $10 \%$ & 15 & $10 \%$ & 17 & $12 \%$ & 18 & $13 \%$ \\
\hline Muy baja & 4 & $3 \%$ & 3 & $2 \%$ & 6 & $4 \%$ & 5 & $3 \%$ & 7 & $5 \%$ \\
\hline Total & 144 & $100 \%$ & 144 & $100 \%$ & 144 & $100 \%$ & 144 & $100 \%$ & 144 & $100 \%$ \\
\hline
\end{tabular}

De los resultados se establece que en los estudiantes del I semestre de la carrera profesional de Psicología de la Universidad Continental existe una tendencia en el nivel adecuado (promedio) de la inteligencia emocional.

En la figura 1 se observa los porcentajes en cada uno de los niveles.

En la tabla 2 se muestra los niveles de inteligencia emocional que tienen los estudiantes que respondieron el inventario, teniendo en cuenta las dimensiones de la inteligencia emocional:

Se observa en los resultados que el mayor porcentaje de estudiantes en cada una de las dimensiones tiene un nivel adecuado. Según Ugarriza (2001) significa que tienen una capacidad emocional promedio.

En la dimensión intrapersonal el nivel adecuado señala que los estudiantes están aceptablemente conformes con la forma de expresar sus sentimientos, se sienten bien acerca de sí mismos y se sienten positivos en lo que están haciendo. Son capaces de expresar sus sentimientos y son independientes y no se doblegan fácilmente cuando desean cristalizar sus ideas. Confían en la realización de sus creencias.

En la dimensión interpersonal el nivel adecuado señala que los estudiantes son medianamente responsables y confiables, que cuentan con habilidades sociales, es decir, que entienden, interactúan y se relacionan aceptablemente y sin mucha dificultad con otros estudiantes o personas en general y les es fácil trabajar en equipo.

En la dimensión adaptabilidad el nivel adecuado nos señala que los estudiantes tienden a ser flexibles, realistas y que comprenden las situaciones problemáticas. Evidencias ser competentes para llegar a soluciones adecuadas, aunque a veces no lo logran. Los estudiantes ubicados en este nivel regularmente salen airosos en el manejo de las dificultades que se les presenta cotidianamente.

En la dimensión manejo de la tensión el nivel adecuado indica que los estudiantes tienen un manejo aceptable de las situaciones que le presionan, como tener tiempos muy cortos para la entrega de trabajos, tener que estudiar cuando tienen problemas familiares - económicos que resolver. Resisten la tensión y presión sin "desmoronarse fácilmente" o "no pierden el control inmediatamente". Son estudiantes que por lo general se muestran relativamente calmados, controlan medianamente sus impulsos y trabajan aceptablemente bajo presión. Pueden realizar, medianamente, sin muchas dificultades actividades que provocan tensión o ansiedad o que involucran cierto riesgo.

En la dimensión estado de ánimo, el nivel adecuado implica que los estudiantes son por lo general alegres, positivos y optimistas, que pueden sin mucho esfuerzo encontrar actividades que le permitan disfrutar de las actividades que realiza cotidianamente. Además de ser un elemento esencial al momento de interactuar con otros individuos, esta dimensión en este nivel motiva e influye medianamente en la solución de problemas y en la tolerancia a la tensión. Además, se observa como un rasgo negativo que en la dimensión estado de ánimo los estudiantes en un $18 \%$ se hallan en los niveles bajo y muy bajo de inteligencia emocional. En las demás dimensiones de la inteligencia emocional, en los niveles bajo y muy bajo, los porcentajes oscilan entre el $12 \%$ y $15 \%$ que son los que tienen que mejorar sus niveles de inteligencia emocional, en todas las dimensiones, debido a que se hallan por debajo del promedio aceptable.

Un dato positivo a destacar es el que corresponde a la dimensión interpersonal, debido a que el $20 \%$ de los estudiantes encuestados se hallan en los niveles alto y muy alto en esta dimensión. A este grupo le caracteriza la facilidad para relacionarse con los demás y realizar trabajos en equipo.

De los datos se puede concluir que si bien en las cinco dimensiones, los estudiantes en mayor porcentaje tienen un nivel adecuado de capacidades emocionales, es relativamente preocupante los porcentajes de estudiantes que hay en los niveles bajo y muy bajo, en todas las dimensiones (o componentes 
de la inteligencia emocional), más aún si se trata de estudiantes de psicología. Esto implica que se tienen que trabajar acciones remediales con ellos en forma urgente.

Además se puede inferir de los resultados que se muestran en la figura 2, que no existen diferencias significativas en las proporciones de estudiantes que se hallan en cada uno de los niveles en cada una de las dimensiones. Pero para probar dicha conjetura,

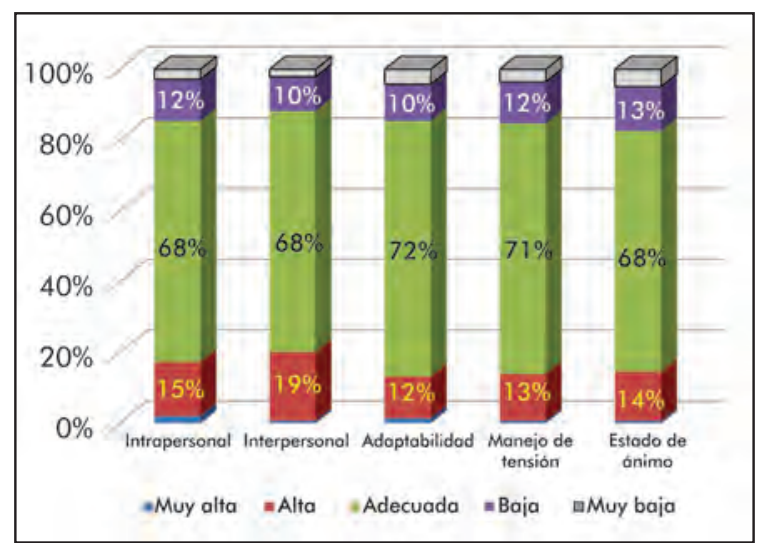

Figura 2. Niveles por dimensiones.

estadísticamente, utilizaremos el estadístico Chi cuadrado.

Se aprecia visualmente que en las cinco dimensiones el nivel predominante es el adecuado y que los porcentajes en los demás niveles son similares proporcionalmente.

La tabla 3 muestra el reporte del procesamiento de datos con el estadístico Chi cuadrado, con el SPSS para establecer si las diferencias que hay en las

Tabla 3

Prueba de homogeneidad - Chi-cuadrado de las proporciones de los niveles por dimensiones de inteligencia emocional.

\begin{tabular}{lccc}
\hline & Valor & gl & $\begin{array}{c}\text { Significación } \\
\text { asintótica } \\
\text { (bilateral) }\end{array}$ \\
\hline $\begin{array}{l}\text { Chi-cuadrado de } \\
\text { Pearson }\end{array}$ & $7,864^{\mathrm{a}}$ & 16 & 0,953 \\
$\begin{array}{l}\text { Razón de } \\
\text { verosimilitud }\end{array}$ & 7,757 & 16 & 0,956 \\
$\begin{array}{l}\text { Asociación lineal } \\
\text { por lineal } \\
\mathrm{N} \text { de casos válidos }\end{array}$ & 2,366 & 1 & 0,124 \\
\hline
\end{tabular}

proporciones de los niveles en las cinco dimensiones, son o no significativas estadísticamente:

De acuerdo al reporte SPSS el $p$ valor $p=0,953$ es mayor que el nivel de significancia de 0,05, por lo que

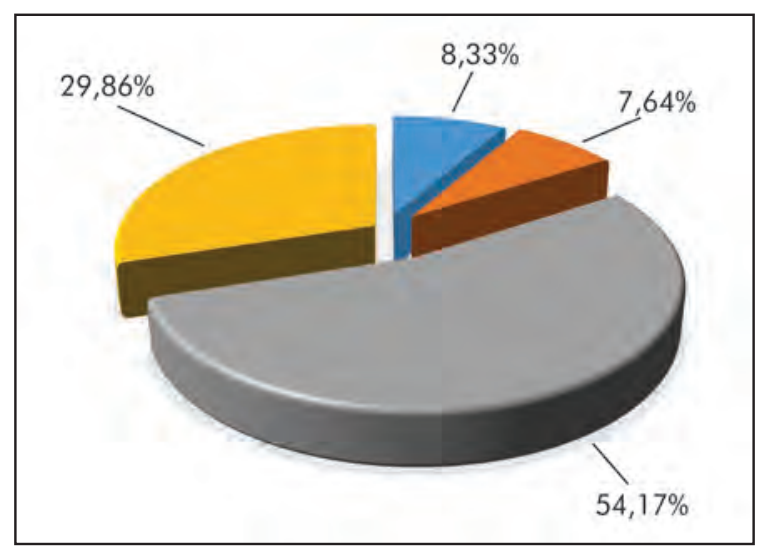

Figura 3. Nivel de rendimiento académico.

se infiere que existen evidencias que las proporciones en cada uno de los niveles de las dimensiones de la inteligencia emocional son homogéneas. Ello corrobora las descripciones de la tabla 3 y figura 2 .

La figura 3 de frecuencias que se muestra permite apreciar que el mayor porcentaje de estudiantes tiene un nivel medio de rendimiento académico.

En la tabla 4 se muestran los estadísticos de tendencia central, variación y forma de la variable rendimiento académico.

Tabla 4

Medidas de tendencia central, variación y forma del rendimiento académico.

\begin{tabular}{llr}
\hline$N^{\circ}$ & \multicolumn{1}{c}{ Estadísticos } & Valores \\
\hline 01 & Media & 14,00 \\
02 & Mediana & 14,00 \\
03 & Moda & 14,00 \\
04 & Desviación estándar & 2,40 \\
05 & Asimetría & $-0,19$ \\
06 & Curtosis & $-0,42$ \\
07 & Mínimo & 8,00 \\
08 & Máximo & 19,00 \\
\hline
\end{tabular}

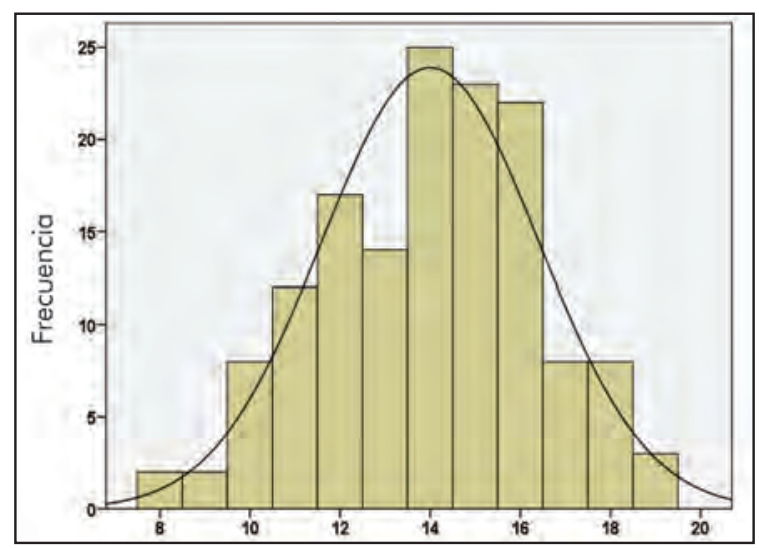

Figura 4. Histograma rendimiento académico. 
Se puede observar que los estadísticos de tendencia central: media mediana y moda tienen un valor de 14 es escala vigesimal, valor que caracteriza al grupo de la muestra de estudiantes del I semestre de la carrera de Psicología de la Universidad Continental $y$, que es medianamente satisfactorio en referencia a su rendimiento académico en la asignatura de Fundamentos de la Psicología. Además se observa que los valores mínimo y máximo tienen valores de 8 y 19 , los mismos que dan un rango de 11 puntos en escala vigesimal. La desviación estándar es de 2,4 que para una escala vigesimal es un valor aceptable, teniendo en cuenta el rango de 11 puntos y la distribución. Los valores de la asimetría y curtosis son negativos cercanos a cero, que indican cola más extendida por la izquierda y achatamiento y que descriptivamente podría ser la de una distribución normal, pero que en la prueba de hipótesis con el estadístico Kolmogorov Smirnof se probó que la variable no tiene distribución normal. En la figura 4 se muestra el histograma con curva normal que reporta el SPSS con los datos de la variable rendimiento académico.

La relación entre inteligencia emocional y rendimiento académico de los estudiantes de la carrera profesional de Psicología de la Universidad Continental se estudia desde la perspectiva de la aplicación de la "rho"

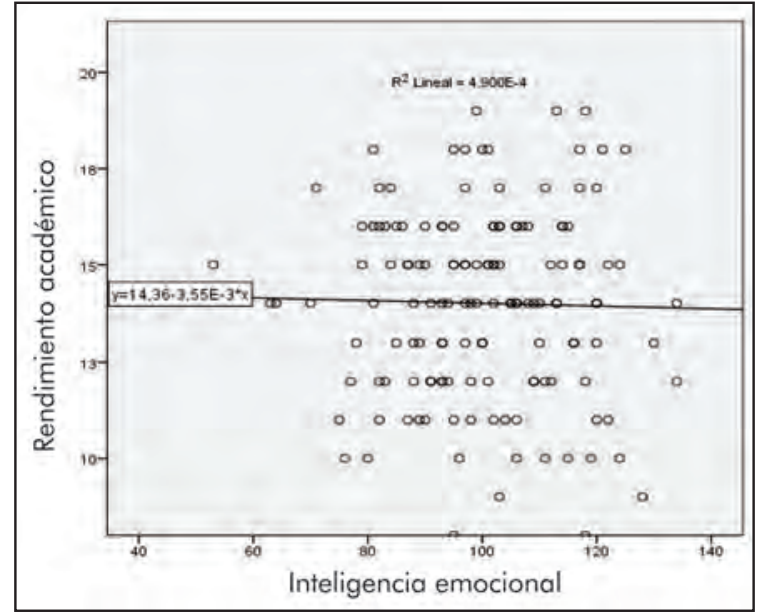

Figura 5. Dispersión de datos.

de Spearman y la " $\dagger$ de student" procesado con el programa estadístico SPSS.

La figura 5, con su línea de ajuste que se muestra, indica que existe una correlación inversa y que la correlación es prácticamente nula, debido a la dispersión de los puntos que se observa. Este análisis descriptivo se comprobará inferencialmente con los

Tabla 5

Correlación, inteligencia emocional y rendimiento académico.

\begin{tabular}{llcc}
\hline Estadísticos & \multicolumn{1}{c}{ Variables } & $\begin{array}{c}\text { Coeficiente de } \\
\text { correlación }\end{array}$ & $\begin{array}{c}\text { Significancia } \\
\text { unilateral }\end{array}$ \\
\hline $\begin{array}{l}\text { Rho de } \\
\text { Spearman }\end{array}$ & Inteligencia emocional & $-0,004$ & 0,479 \\
\hline
\end{tabular}

Tabla 6

Correlación, intrapersonal y rendimiento académico.

\begin{tabular}{llcc}
\hline Estadísticos & \multicolumn{1}{c}{ Variables } & $\begin{array}{c}\text { Coeficiente de } \\
\text { correlación }\end{array}$ & $\begin{array}{c}\text { Significancia } \\
\text { unilateral }\end{array}$ \\
\hline $\begin{array}{l}\text { Rho de } \\
\text { Spearman }\end{array}$ & Intrapersonal & $-0,029$ & 0,366 \\
\hline
\end{tabular}

Tabla 7

Correlación, interpersonal y rendimiento académico.

\begin{tabular}{llcc}
\hline Estadísticos & \multicolumn{1}{c}{ Variables } & $\begin{array}{c}\text { Coeficiente de } \\
\text { correlación }\end{array}$ & $\begin{array}{c}\text { Significancia } \\
\text { unilateral }\end{array}$ \\
\hline $\begin{array}{l}\text { Rho de } \\
\text { Spearman }\end{array}$ & Interpersonal & 0,009 & 0,458 \\
\hline
\end{tabular}

Tabla 8

Correlación, adaptabilidad y rendimiento académico.

\begin{tabular}{llcc}
\hline Estadísticos & \multicolumn{1}{c}{ Variables } & $\begin{array}{c}\text { Coeficiente de } \\
\text { correlación }\end{array}$ & $\begin{array}{c}\text { Significancia } \\
\text { unilateral }\end{array}$ \\
\hline $\begin{array}{l}\text { Rho de } \\
\text { Spearman }\end{array}$ & Adaptabilidad & $-0,057$ & 0,249 \\
\hline
\end{tabular}


Tabla 9

Correlación, manejo de tensión y rendimiento académico.

\begin{tabular}{llcc}
\hline Estadísticos & \multicolumn{1}{c}{ Variables } & $\begin{array}{c}\text { Coeficiente de } \\
\text { correlación }\end{array}$ & $\begin{array}{c}\text { Significancia } \\
\text { unilateral }\end{array}$ \\
\hline $\begin{array}{l}\text { Rho de } \\
\text { Spearman }\end{array}$ & $\begin{array}{l}\text { Manejo de tensión } \\
\text { Rendimiento académico }\end{array}$ & $-0,021$ & 0,404 \\
\hline
\end{tabular}

Tabla 10

Correlación, estado de ánimo y rendimiento académico.

\begin{tabular}{llcc}
\hline Estadísticos & \multicolumn{1}{c}{ Variables } & $\begin{array}{c}\text { Coeficiente de } \\
\text { correlación }\end{array}$ & $\begin{array}{c}\text { Significancia } \\
\text { unilateral }\end{array}$ \\
\hline $\begin{array}{l}\text { Rho de } \\
\text { Spearman }\end{array}$ & $\begin{array}{l}\text { Estado de ánimo } \\
\text { Rendimiento académico }\end{array}$ & 0,066 & 0,215 \\
\hline
\end{tabular}

resultados de la prueba rho de Spearman.

Según Cancela (2010), el reporte del SPSS rho $=$ $-0,004$ nos indica que existe una correlación inversa y prácticamente nula, es decir que no existe correlación.

El "p valor" que se muestra en la tabla $p=0,479$ es mayor al nivel de significancia de 0,05 y de acuerdo a nuestra regla de decisión se acepta la hipótesis nula que dice: No existe relación directa entre las variables Inteligencia emocional y Rendimiento académico en la asignatura de Fundamentos de la Psicología en los estudiantes del I semestre de la carrera profesional de Psicología de la Universidad Continental.

Según Cancela (2010), el reporte del SPSS rho = $-0,029$ nos indica que existe una correlación inversa y prácticamente nula, es decir que no existe correlación.

El "p valor" que se muestra en la tabla $p=0,366$ es mayor al nivel de significancia de 0,05 y de acuerdo a nuestra regla de decisión se acepta la hipótesis nula que dice: No existe relación directa entre la dimensión intrapersonal de la variable Inteligencia emocional y el rendimiento académico en la asignatura de Fundamentos de la Psicología en los estudiantes del I semestre de la carrera de Psicología de la Universidad Continental.

Según Cancela (2010), el reporte del SPSS rho $=$ 0,009 nos indica que existe una correlación inversa y prácticamente nula, es decir que no existe correlación.

El " $p$ valor" que se muestra en la tabla $p=0,458$ es mayor al nivel de significancia de 0,05 y de acuerdo a nuestra regla de decisión se acepta la hipótesis nula que dice: No existe relación directa entre la dimensión interpersonal de la variable Inteligencia emocional y el rendimiento académico en la asignatura de Fundamentos de la Psicología en los estudiantes del I semestre de la carrera de Psicología de la Universidad Continental.
Según Cancela (2010), el reporte del SPSS rho $=$ $-0,057$ nos indica que existe una correlación inversa y prácticamente nula, es decir que no existe correlación.

El " $p$ valor" que se muestra en la tabla $p=0,249$ es mayor al nivel de significancia de 0,05 y de acuerdo a nuestra regla de decisión se acepta la hipótesis nula que dice: No existe relación directa entre la dimensión adaptabilidad de la variable Inteligencia emocional y el rendimiento académico en la asignatura de Fundamentos de la Psicología en los estudiantes del I semestre de la carrera de Psicología de la Universidad Continental.

Según Cancela (2010), el reporte del SPSS rho = $-0,021$ nos indica que existe una correlación inversa y prácticamente nula, es decir que no existe correlación. El " $p$ valor" que se muestra en la tabla $p=0,404$ es mayor al nivel de significancia de 0,05 y de acuerdo a nuestra regla de decisión se acepta la hipótesis nula que dice: No existe relación directa entre la dimensión manejo de tensión de la variable Inteligencia emocional y el rendimiento académico en la asignatura de Fundamentos de la Psicología en los estudiantes del I semestre de la carrera de Psicología de la Universidad Continental.

Según Cancela (2010), el reporte del SPSS rho = 0,066 nos indica que existe una correlación inversa y prácticamente nula, es decir que no existe correlación.

El " $p$ valor" que se muestra en la tabla $p=0,215$ es mayor al nivel de significancia de 0,05 y de acuerdo a nuestra regla de decisión se acepta la hipótesis nula que dice: No existe relación directa entre la dimensión estado de ánimo de la variable Inteligencia emocional y el rendimiento académico de la asignatura de Fundamentos de Psicología en los estudiantes del I semestre de la carrera de Psicología de la Universidad Continental. 


\section{DISCUSIÓN}

Jiménez Morales y López-Zafra (2009), en su artículo: "Inteligencia emocional y rendimiento escolar: estado actual de la cuestión", manifiesta que luego de muchos años de investigación, que la relación existente entre inteligencia emocional y rendimiento académico, mostró que los resultados son inconsistentes debido a varios factores: definición de inteligencia emocional, operacionalización del constructo y la metodología de los estudios. En este mismo estudio, se menciona que la validez predictiva de los test cognitivos respecto al pronóstico del éxito posterior ha hecho que los investigadores dirijan su atención a otras variables como la inteligencia emocional.

Existen dos modelos que teorizan lo que es la inteligencia emocional: el modelo de Mayer y Salovey (1997), es un modelo que entiende la IE, como una inteligencia genuina que se basa en el uso de adaptativo de las emociones para adaptarse al medio ambiente. El modelo "mixto" de Bar-On (1997), tiene una visión más difusa ya que este modelo se centra en los rasgos estables de comportamiento y variables de personalidad.

Jiménez Morales y López-Zafra (2009), manifestó en su artículo mencionado que los aspectos afectivos, como el bienestar o la satisfacción son una constante en el proceso enseñanza-aprendizaje. Este artículo también apunta que la relación entre éxito académico, y competencia emocional han aportado resultados inconsistentes y contradictorios. Esta afirmación coincide consistentemente con la hipótesis nula y los resultados obtenidos en la presente investigación.

En referencia a lo anterior, según Paul Eckman (2003), en su libro "El rostro de las emociones", respecto a los resultados de su investigación sobre las emociones y las ciencias del comportamiento en general manifiesta: "Lo que hallé fue exactamente lo opuesto de lo que pensaba que iba a descubrir. Es lo ideal. Los hallazgos de las ciencias del comportamiento son más creíbles, cuando, más que confirmar las expectativas de los científicos, las contradicen. En la mayor parte de campos de estudio científico ocurre justamente lo contrario: el crédito que sus hallazgos merecen aumenta si ya habían sido pronosticados. Ello es debido a que la posibilidad de desviación o error queda sujeta a comprobación por esa tradición de que unos científicos reproduzcan los experimentos realizados por otros para ver si llegan a los mismos resultados. Desgraciadamente, en las ciencias del comportamiento dicha tradición es inexistente. Es muy raro que un experimento se repita, sea por científicos que originalmente lo realizaron o por otros. Careciendo de dicha garantía, los científicos del comportamiento resultan más propensos a encontrar inconscientemente sólo lo que desean hallar.
En ese sentido la hipótesis inicial de la presente investigación, fue que existe relación entre la inteligencia emocional y el rendimiento académico en la asignatura de Fundamentos de la Psicología de los estudiantes del primer semestre de la Escuela Académico Profesional de Psicología de la Universidad Continental. Los resultados tanto de las investigaciones consultadas como los resultados obtenidos en esta investigación corroboran claramente lo mencionado anteriormente.

Según la tabla 5, se observa en la tabla $p=0,479$ es mayor al nivel de 0,05 y de acuerdo a nuestra regla de decisión se acepta la hipótesis nula que dice: No existe relación directa entre las variables Inteligencia emocional y Rendimiento académico en la asignatura de Fundamentos de la Psicología en los estudiantes del I semestre de la carrera profesional de Psicología de la Universidad Continental. Este resultado que se repetirá en todos los subcomponentes está en correspondencia con estudios antes realizados que encontraron que no existe relación directa entre la IE y el rendimiento académico.

\section{REFERENCIAS BIBLIOGRÁFICAS}

Alarcón, R. (2000). La Historia de la Psicología en el Perú. De la Colonia a la República. Lima: UPRP.

ANR (2006). La Carrera de Psicología en el Perú. Lima: Dirección General de Investigación y Acreditación Universitaria.

Bar-On, R. (1997). The Emotional Intelligence Inventory (EQ-i): Technical manual . Toronto: Multi-Health Systems.

Cancela Gordillo, R. (2010). Metodología de la Investigación Educativa: Investigación ex Post Facto. Universidad Autónoma de Madrid, 1-19.

Ekman, P. (2003). El rostro de las emociones. Barcelona: RBA.

Extremera, N., y Rey, L. (2007). Inteligencia emocional y su relación con sus niveles de Burnout, engagement y estrés en estudiantes universitarios. Revista de Educación, 239-256.

Jiménez Morales, M., y López-Zafra, E. (2009). Inteligencia emocional y rendimiento escolar: estado actual de la cuestión. Revista Latinoamericana de Psicología, vol.41, 69-79.

Mayer, J., y Salovey, P. (1997). Emotional Intelligence. Imagination, Cognition, and Personality, 185-225.

Páez, M., y Castaño. (2015). Inteligencia emocional y rendimiento académico en estudiantes universitarios. Psicología desde el Caribe. Universidad del Norte. Vol. 32, 268-285.

Ugarriza, N., yL, P. (2001). Adaptación y estandarización del inventario de inteligencia emocional de Bar-On ICE:NA, en niños y adolescentes. Lima: Amigo. 